\title{
Erratum to: The Effect of Substitution of As for Ga on the Topological Phase and Structural, Electronic and Magnetic Properties of $\mathrm{Mn}_{2} \mathrm{ZrGa}$ Heusler Alloy
}

\author{
Ahmad Amirabadizadeh $^{1}$ - Seyyed Amir Abbas Emami ${ }^{1}$. Zahra Nourbakhsh ${ }^{2}$. \\ Seyyed Mojtaba Alavi Sadr ${ }^{3}$. Seyyed Madhy Baizaee ${ }^{4}$
}

Published online: 13 December 2016

(c) Springer Science+Business Media New York 2016

Erratum to: J Supercond Nov Magn (2016)

DOI 10.1007/s10948-016-3884-5

The original version of this article unfortunately contained a mistake. The symbol As for Arsenic was incorrectly presented as "as".

The original article was corrected.

The online version of the original article can be found at http://dx.doi.org/10.1007/s10948-016-3884-5.

\footnotetext{
Ahmad Amirabadizadeh

aamirabadizade@birjand.ac.ir

1 Department of Physics, Faculty of Science, University of Birjand, P.O. Box: 97175-615, Birjand, Iran

2 Physics Department, Faculty of Science, University of Isfahan, Isfahan, Iran

3 Department of Basic Sciences, Birjand University of Technology, Birjand, Iran

4 Department of Physics, Vali-e-Asr University of Rafsanjan, Rafsanjan, Iran
} 